\title{
High Directivity Horn Antenna of Metamaterial in Terahertz
}

\author{
Xiangjin Quan, Shiquan Zhang, Hui Li \\ Engineering University of CAPF, Xi'an, 710086, China
}

Keywords: Electromagnetic metamaterials; THz; Triangular unit's superstrate; high directivity antenna.

\begin{abstract}
A triangular metamaterial structure unit is presented and the effective permittivity and permeability parameters are retrieved from the transmission and reflection data. Simulation results show that the combined unit exhibits left-handed passband in terahertz (THz). Triangular electromagnetic metamaterial is applied to conventional $\mathrm{THz}$ and the characteristics of zero refractive index are used to produce horn antennas superstrate for directional radiation of electromagnetic, so as to improve the antenna directivity and gain. The experimental results show that antenna with triangular magnetic metamaterials results in weaken backward wave radiation, enhanced forward radiation efficiency enhancement and the directivity factor increased by 46.86 and the gain is increased by $2.77 \mathrm{~dB}$.
\end{abstract}

\section{Introduction}

Following microwave band and infrared band, terahertz $(\mathrm{THz})$ band becomes another research hot spot but this research has been very limited due to its particular band position. In recent years, with the higher requirements for communication bandwidth, actor surveillance, electronic countermeasure, inspection imaging, etc., the THz band gradually wins people's attention. However, the research in this band is weaker than that of microwave band and infrared band, most of which is still in theory phase while the research on actual application is little. In terms of antenna in particular, to make use of the advantages of the $\mathrm{THz}$ band, the application of antenna is inseparable in both the establishment of communication system and the realization of radar detection. Since the wavelength of $\mathrm{THz}$ is small and the electromagnetic loss in the wireless transmission is large, how to overcome this defect and make high direction antenna become very significant. However, in the application of satellite and radar, the horn antenna has to deal with the restriction between the gain and directivity and the dimensions. Traditionally, it is to add the curved lens at the calibre of the antenna. However, since the focusing performance is very sensitive to the curvature of the curved surface, the traditional method is faced with many difficulties in real application. Since 1968 when the concept of electromagnetic metamaterial was put forward [1], its various particular characteristics have won widespread attention among which one important direction is the research on the improvement of the antenna performance. In microwave band, the electromagnetic metamaterial is widely used to improve the antenna performance [2-6], C. G. Parasol, et al. used horn antenna to receive and send the electromagnetic wave, and verified its negative refraction performance in open space through prism refraction experiment. But there is still little optimization about the THz band antenna.

One horn antenna working in the THz band is designed. Metamaterials structure unit is used to optimize its performance and triangular metamaterials structure unit is added to the calibre of the horn antenna so as to form plane lens to converge the wave beams of the antenna. The influence of metamaterials unit on the performance of antenna is discussed in theory and the experiment. The results show that the horn antenna with metamaterials unit has significant improvement in gain, directivity coefficient and radiation efficiency comparing normal microwave band antenna.

Permittivity and permeability of the metamaterials can be controlled by artificial parameter adjustment. Therefore, the particular optical characteristics of the metamaterials can be applied to realize the focusing of the slab lens so as to improve the gain and the directivity of the antenna. Hence, two ways are used, one is to use metamaterials unit to make antenna cover layer and the other is to use the metamaterials unit to add antenna substrate. 
The metamaterials which has near-zero refraction index are used as the covering layer of the antenna to improve the antenna directivity, and gathers together the transmission direction of the electromagnetic wave to realize the oriented transmission. Assume that the TE electromagnetic wave enters the air dielectric from the metamaterials, the wave vector, $\vec{k}=\hat{x} k_{x}+\hat{z} k_{z}$ is in the plane of $x-z$. Taking the interface of metamaterials and air dielectric as the axis $z$ and the incidence direction of the wave is the direction $x$, i.e. the wave is from the half plane of metamaterials of $x<0$ to the half plan of air dielectric of $x>0$, when the main axis of metamaterials is parallel to the interface, we can represent the electromagnetic parameter of the metamaterials as:

$$
\begin{aligned}
& \bar{\varepsilon}=\varepsilon_{0} \overline{\overline{\varepsilon_{r}}}=\varepsilon_{0} \operatorname{diag}\left[\varepsilon_{r x} ; \varepsilon_{r y} ; \varepsilon_{r z}\right] \\
& =\overline{\bar{\mu}}=\mu_{0} \overline{\overline{\mu_{r}}}=\mu_{0} \operatorname{diag}\left[\mu_{r x} ; \mu_{r y} ; \mu_{r z}\right]
\end{aligned}
$$

As for $T E$ wave, the polarization direction of electric field is $y$, i.e. $k_{y}=0$, then

$$
\frac{k_{x}^{2}}{\mu_{r z} \varepsilon_{r y}}+\frac{k_{z}^{2}}{\mu_{r x} \varepsilon_{r y}}=k_{0}^{2}
$$

The two components of refractive index are $n_{x}=\sqrt{\mu_{r z} \varepsilon_{r y}}$ and $n_{z}=\sqrt{\mu_{r x} \varepsilon_{r y}}$. When $\left|\mu_{r x}\right|<<1, n_{z} \approx 0$ while $n_{x} \neq 0$, then the transmission of electromagnetic wave meets $k_{z} \approx 0$ and $k_{x}>0$. Since this transmission must meet the phase matching conditions, the interface between metamaterials and air dielectric is axis $z$, the radiation direction of electromagnetic wave gathers together nearby the normal vector between the dielectric and air interface, as shown in Fig.1.

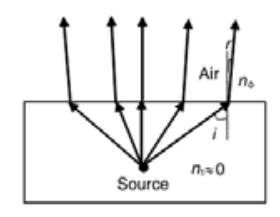

Fig.1 The principle of metamaterials as the cover layer to realize the electromagnetic wave orientation

\section{Experiment result analysis}

\section{Design of Triangular metamaterials unit}

Fig.2 shows the physical dimensions of triangular metamaterial unit designed in this paper. These two metamaterial units both have three layers of dielectrics vertical to axis $\mathrm{z}$. the electromagnetic wave is perpendicular to the structure unit. The two planes parallel to the axis $\mathrm{z}$ are set to be the excitation of wave ports and the load impedance is $50 \Omega$. The two planes parallel to the axis $\mathrm{X}$ is set to be PEC. Anti-parallel current will be induced on metal structure of the two faces of the substrate with the action of the field to realize the magnetic resonance. The metal rod in the structure will produce electric resonance for electric field induction. The resonance frequency of the metamaterials unit can be adjusted by optimizing of the dimension parameters.

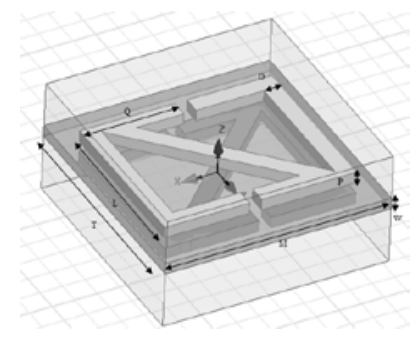

Fig.2 Triangular metamaterial unit structure

The two sides of triangular metamaterial unit structure are printed on the two sides of the $1.6 \mathrm{~mm}$, thick of FR4 epoxy resin board with the permittivity $\varepsilon_{r}=4.4$. The geometrical parameters are $T=600 u m, W=20 u m, M=600 u m, P=20 u m, L=280 u m, D=30 u m, Q=110 u m$. 
Near-zero refractive index of the metamaterials is applied when the triangular metamaterials unit is used to make cover layer. Three-dimensional electromagnetic simulation software a soft HFSS based on Finite Element Method of magnetic field is used in the experiment software and ACER computer is used as the hardware for calculation. The calculation boundary of simulation is $8 \times 8 \times 8 \mathrm{~mm}^{3}$.

The Fig.3 (a) shows the S parameter of the Z-type metamaterials unit obtained through HFSS software $\left(S_{11}\right.$ is the transmission parameter, $S_{12}$ is the reflection parameter). Fig.3 (b) shows the permeability of the $\mathrm{Z}$ type metamaterial unit after being treated with Mat lab using NRW ${ }^{[17]}$ algorithm. The results show that near-zero refractive index can be obtained at $0.34 \mathrm{THz}$.

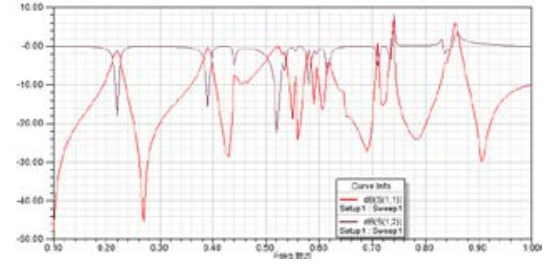

(a)

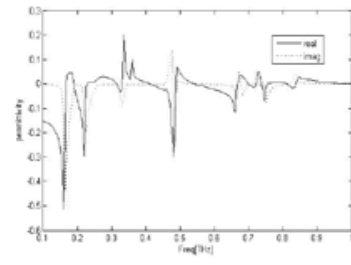

(b)

Fig.3 The equivalent permeability of parameter (b) of transmssion curve $S$

\section{Horn antenna design based on triangular metamaterials unit}

Rectangular waveguide with the model of WR-3 is used in horn antenna. The operation frequency range in this model is $217 \mathrm{GHz}-370 \mathrm{GHz}$, the dimensions are $0.8636 \mathrm{~mm} \times 0.4318 \mathrm{~mm}$ and the wall thickness of waveguide is $0.76 \mathrm{~mm}$. Coaxial feed method is used. Since the computer performance for the experiment is limited, the antenna gain after deign required by the experiment is no less than $20 \mathrm{~dB}$ and the bandwidth is no less than $20 \%$ and the working centre frequency is $0.34 \mathrm{THz}$. After calculation, the width and the length of the horn calibre is $A=3.89 \mathrm{~mm}$ and $B=3.17 \mathrm{~mm}$ respectively, the waveguide length is $W=1.1 \mathrm{~mm}$, and the length of the horn is $P=5 \mathrm{~mm}$. The radius of the external conductor of coaxial line is set to be $0.012 \mathrm{~mm}$ and the length is $0.06 \mathrm{~mm}$, and the radius of the internal conductor is set to be $0.005 \mathrm{~mm}$. The length is the half of that of narrow side of the waveguide.

The filling of horn antenna in the dielectric slab of metamaterial unit is to converge the radiation wave. Therefore, when the metamaterial unit is filled at the calibre of the horn antenna and the HFSS is used for simulation, the software is very sensitive to the model size. The horn of horn antenna is similar to trapezoidal. The rectangular metamaterial unit established directly cannot be filled directly, so we place the metamaterial unit at the front end of the horn for simulation. The model is shown in Fig.4.

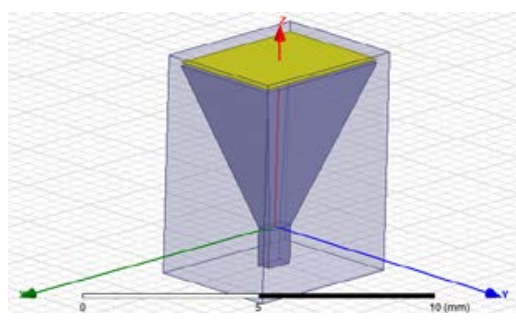

\section{Return loss}

Fig.4 filling of horn antenna of metamaterial

The return loss is obtained for horn antenna of metamaterial unit through experiment simulation as shown in Fig.5. As shown in the figure, the maximum value of return loss of the antenna filled with dielectric slab of the metamaterial occurs at the place where working centre frequency of the original horn antenna is $0.34 \mathrm{THz}$ and the loss value is $-30.37 \mathrm{~dB}$. Compared with horn antenna without filling of dielectric slab, the peak value of the return loss is reduced by $2.52 \mathrm{~dB}$. Therefore, the filling of dielectric slab does not affect the matching of the antenna but improves the matching. 


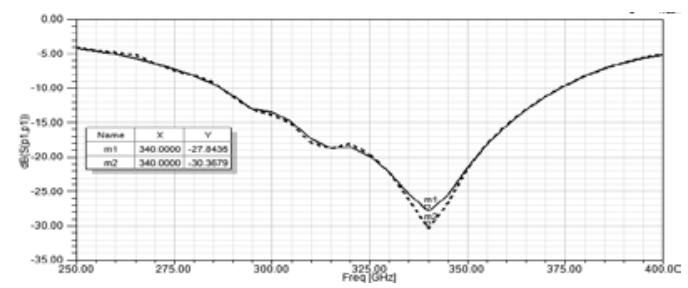

\section{Gain directivity figure}

Fig.5 Return loss of horn antenna with metamaterial dielectric filled

Fig.6 shows the gain directive figure of horn antenna filled with dielectric slab of metamaterial unit. The dotted line represents the antenna after metamaterial unit is filled, and the solid line represents the original horn antenna. Figure (a) is the directivity figure of $P h i=0^{\circ}$, i.e. plane E and figure (b) is the directivity figure of $\mathrm{Phi}=90^{\circ}$ and i.e. plane $\mathrm{H}$. As shown in the figure, antenna gain filled with metamaterial is $22.67 \mathrm{~dB}$, an increase of $2.77 \mathrm{~dB}$ than antenna without cover layer, which is an increase of $13.85 \%$. Meanwhile, compared with normal horn antenna, the width (-3dB) of half power wave beam for the plane $\mathrm{E}$ and plane $\mathrm{H}$ is shrunk by $2.5^{\circ}$ and $0.8^{\circ}$, respectively, compared with normal horn antenna.

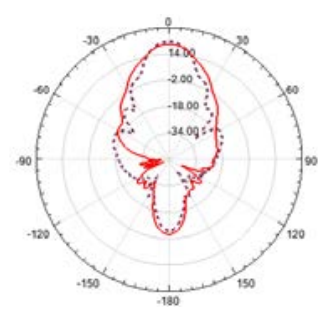

(a) Plane $E$

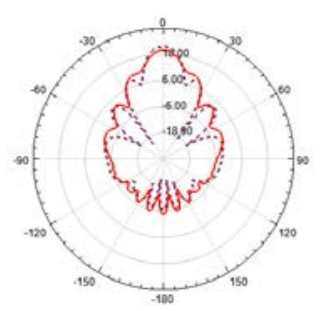

(b) Plane $H$

\section{Directivity coefficient}

Fig.6 Horn antenna directivity with metamaterials unit filled

The directivity coefficient of horn antenna filled with metamaterials unit is shown in Fig.7. As shown in this figure, the directivity coefficient of antenna filled with metamaterial unit is 145.63 , which is an increase of 46.86 , i.e. $47.44 \%$ compared with the antenna without metamaterial unit. With the same parameters, the height of normal horn antenna has to be increased by 1.5 times to reach this directivity coefficient.

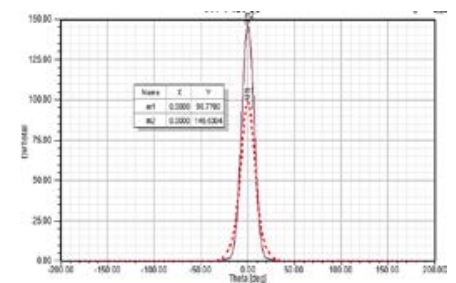

Fig.7 Directivity coefficient of horn antenna filled with metamaterial

As shown in above results, comparing the return loss of the normal $\mathrm{THz}$ horn antenna with the horn antenna filled with triangular metamaterials units, filling metamaterials units on the basis of normal horn antenna does not change the centre resonance frequency of original antenna and the return loss is further reduced. And the adding of triangular metamaterials units increases the antenna gain by $2.77 d B$ and the directivity coefficient is increased by 46.86 and various performance parameters of the antenna are enhanced.

\section{Conclusion}

Triangular metamaterial unit is loaded to the horn antenna working in $\mathrm{THz}$ band in this paper, zero refractive index is used to make cover layer of the antenna to realize the oriented radiation of the electromagnetic wave, resulting in the reduction of the energy loss of backward wave and the improvement of the antenna directivity coefficient and gain. Experiment results show that the return 
loss at the working centre frequency is reduced by $2.77 \mathrm{~dB}$ and the matching of antenna impedance is optimized, the antenna gain is increased by $2.77 d B$, the width of half power wave lobe at $P h i=0^{\circ}$ and $P h i=90^{\circ}$ is reduced by $2.5^{\circ}$ and by $\mathbf{O} .8^{\circ}$ respectively and the directivity coefficient is increased by 46.86. Filling the calibre with dielectric slab of metamaterial unit not only improves the orientated radiation ability of the antenna, but also increases the radiation gain and efficiency of the antenna. The idea of combining metamaterial unit with $\mathrm{THz}$ horn breaks the limit that the performance parameters of the horn antenna are improved by increasing the size of itself, which has certain enlightenment significance.

\section{Acknowledgment}

This work is supported by the National Nature Science Foundation of China (Grant No.61072034), the National Nature Science Foundation of China (Grant No.Q1302316), the National Nature Science Foundation of China (Grant No.61265006) and the Basic Research Project of Engineering University of CAPF (Grant No.WJY201404).

\section{References}

[1] Vassalage V G. The electrodynamics of substances with simultaneously negative values of $\varepsilon$ and $\mu$ [J]. Soviet Physics Usp, 1968, 10(4): p.509-514.

[2] Y ANG R, XIE Y, W ANG P, et al. Characteristics of millimetre wave micro strip antenna with left-handed materials substrates [J]. Apple Phis Lett, 2006, 89: 064108(1-3)

[3] MOSALLAEI H, SARABANDI K. Antenna miniaturization and bandwidth enhancement using a reactive impedance substrate [J]. IEEE Trans Antennas Protag, 2004, 52(9):p. 2403-2414.

[4] LIU Y H, ZHAO X P. Investigation of anisotropic negative permeability medium cover for patch antenna [J]. IET Microwaves, Antennas and Propagation, 2008. 7(2): p.737-744.

[5] LIU Y H, ZHAO X P. Enhanced patch antenna performances using dendritic structure metamaterials [J]. Microwave and Optical Technology Letters, 2009, 51(7): p. 1732-1738.

[6] TANG H F, ZHAO X P. Centre-fed circular epsilon-negative zeroth-order resonator antenna [J]. Microwave and Optical Technology Letters, 2009, 51(10): p.2423-2428.

[7] Parazzoli C G, Greegor R B, Li K, et al. Experimental Verification and Simulation of Negative Index of Refraction Using Snell’s Law[J]. Physical Review Letters, 2003, 90: p.107401+4. 